\title{
NOTAS SOBRE A PESCA DA CAVALA E DA SERRA NO CEARÁ - DADOS DE $1970^{(1)}$
}

\author{
Raimundo Saraiva da Costa - Melquíades Pinto Paiva
}

Laboratório de Ciências do Mar
Universidade Federal do Ceará
Fortaleza - Ceará - Brasil

Com o presente trabalho, o Laboratório de Ciências do Mar da Universidade Federal do Ceará dá prosseguimento à série de estudos sobre a pesca da cavala, Scomberomorus cavalla (Cuvier), e da serra, Scomberomorus maculatus (Mitchill), efetuada em águas costeiras do Estado do Ceará, Brasil.

Ressaltamos que durante o ano de 1970 a pesca desses peixes foi conduzida dentro do padrão tradicional, com embarcações, artes e métodos primitivos.

\section{MATERIAL}

O material que serve de fundamento a este trabalho foi capturado em frente ao município de Fortaleza, tendo sido desembarcado na praia de Mucuripe. Corresponde a amostras de 238 dias de pescarias, abrangendo o período de janeiro a dezembro de 1970 . As pescarias foram realizadas por jangadas e alguns botes motorizados, operando com linhas de corso. Foram amostradas 4.616 cavalas e 2.556 serras (tabelas I, II e III). Também, controlalamos 2.301 pescarias de jangadas, realizadas dentro do padrão referido, na mesma área $\mathrm{e}$ durante o período de janeiro a dezembro de 1970 (tabela VI) .

\section{MÉTODO}

Em cada amostra procuramos incluir o máximo possível de indivíduos de ambas as espécies, desembarcados na praia de Mucuripe, e registrados à tarde, após a chegada das embarcações que volviam da faina diária de pesca.

(1) - Trabalho realizado em decorrência de convênios celebrados com a Superintendência do Desenvolvimento do Nordeste (SUDENE) e a Superintendência do Desenvolvimento da Pesca (SUDEPE).
Durante a obtenção das amostras, para cada indivíduo, anotamos apenas a espécie e o comprimento zoológico. Por serem espécimeris de alto valor comercial e de demanda imediata, o conhecimento dos sexos estava acima das nossas possibilidades, uma vez que eram vendidos antes mesmo da evisceração.

Consideramos como comprimento zoológico (fork length) a distância entre o extremo anterior da cabeça e o extremo posterior dos raios medianos da caudal, estando o animal estendido lateralmente sobre uma superfície plana.

Os comprimentos zoológicos dos indivíduos amostrados, de ambas as espécies, foram agrupados, segundo as variações apresentadas, nos respectivos grupos de idades. A aplicação deste procedimento baseia-se nos trabalhos de Nomura \& Rodrigues (1967) e Nomura

\section{T A B E L A I}

Número de amostras e de indivíduos amostrados, correspondentes a pescarias de cavala, Scomberomorus cavalla (Cuvier), e de serra, Scomberomorus maculatus (Mitchill), realizadas em frente ao município de Fortaleza (Estado do Ceará - Brasil), durante o período de janeiro a dezembro de 1970 .

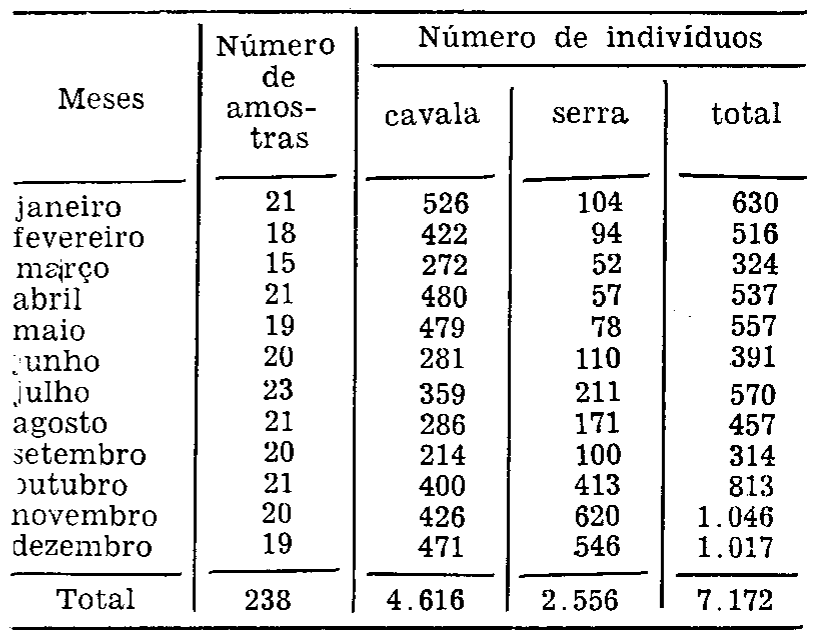


T A B E L A I I

Freqüéncias de cavala, Scomberomorus cavalla (Cuvier), por classes de idades, trimestres e ano. Material capturado em frente ao município de Fortaleza (Dstado do Ceará - Brasil), durante o período de janeiro a dezembro de 1970 .

\begin{tabular}{|c|c|c|c|c|c|c|}
\hline \multirow{2}{*}{$\begin{array}{l}\text { Idades } \\
\text { (anos) }\end{array}$} & \multirow{2}{*}{$\begin{array}{l}\text { Variação do compri- } \\
\text { mento zoológico } \\
(\mathrm{cm})\end{array}$} & \multicolumn{4}{|c|}{ Trimestres } & \multirow{2}{*}{ Ano } \\
\hline & & I & II & III & IV & \\
\hline $\begin{array}{l}\text { I } \\
\text { II } \\
\text { III } \\
\text { IV } \\
\text { V } \\
\text { VI } \\
\text { VII } \\
\text { VIIr } \\
\text { IX } \\
\text { X } \\
\text { XI } \\
\text { XII }\end{array}$ & $\begin{array}{r}30,2 \\
30,3-43,4 \\
43,5-56,5 \\
56,6=67,6 \\
67,7-77,0 \\
77,1-85,5 \\
85,6-92,8 \\
92,9-99,0 \\
99,1-104,5 \\
104,6-109,4 \\
109-5=113,5 \\
=113,6\end{array}$ & $\begin{array}{r}0,1 \\
12,0 \\
25,8 \\
24,6 \\
20,8 \\
11,5 \\
3,9 \\
0,7 \\
0,2 \\
0,2 \\
0,2\end{array}$ & $\begin{array}{c}-\bar{z} \\
\overline{5,5} \\
54,2 \\
19,2 \\
12,1 \\
5,3 \\
2,0 \\
0,6 \\
0,6 \\
0,2 \\
0,3\end{array}$ & $\begin{array}{r}- \\
\overline{6} \\
6,2 \\
47,2 \\
23,7 \\
12,0 \\
6,4 \\
2,8 \\
0,1 \\
0,8 \\
0,1 \\
0,7\end{array}$ & $\begin{array}{r}\overline{0,2} \\
6,8 \\
22,9 \\
39,4 \\
16,2 \\
10,2 \\
3,0 \\
0,6 \\
0,2 \\
\overline{0,5}\end{array}$ & $\begin{array}{r} \\
0,1 \\
7,7 \\
36,6 \\
27,2 \\
15,5 \\
8,5 \\
2,9 \\
0,5 \\
0,4 \\
0,1 \\
0,5\end{array}$ \\
\hline \multirow{2}{*}{ Total } & n. ${ }^{\circ}$ & 1.220 & 1.240 & 859 & 1.297 & 4.616 \\
\hline & $\%$ & 100,0 & 100,0 & 100,0 & 100,0 & 100,0 \\
\hline
\end{tabular}

T A B E L A I I I

Freqüências de serra, Scomberomorus maculatus (Mitchill), por classes de idades, trimestres e ano. Material capturado em frente ao município de Fortaleza (Estado do Ceará - Brasil), durante o período de janeiro a dezembro de 1970 .

\begin{tabular}{|c|c|c|c|c|c|c|}
\hline \multirow{2}{*}{$\begin{array}{l}\text { Idades } \\
\text { (anos) }\end{array}$} & \multirow{2}{*}{$\begin{array}{l}\text { Variação do compri- } \\
\text { mento zoológico } \\
(\mathrm{cm})\end{array}$} & \multicolumn{4}{|c|}{ Trimestres } & \multirow{2}{*}{ Ano } \\
\hline & & I & II & III & IV & \\
\hline I & 25,6 & - & - & - & - & - \\
\hline II & $25,7-37,9$ & 0,4 & 2,0 & 3,7 & 0,4 & $\overline{1,2}$ \\
\hline III & $38,0-45,8$ & 18,8 & 38,0 & 24,5 & 10,7 & 16,7 \\
\hline IV & $45,9-56,3$ & 39,2 & 42,5 & 39,6 & 43,3 & 42,1 \\
\hline $\mathrm{V}$ & $56,4-59,7$ & 11,6 & 4,1 & 10,0 & 15,9 & 13,2 \\
\hline VI & $59,8-64,6$ & 6,4 & 6,9 & 10,8 & 14,4 & 12,2 \\
\hline VII & $64,7-69,1$ & 11,6 & 4,1 & 5,2 & 8,7 & 7,9 \\
\hline VIII & $69,2-74,8$ & 7,6 & 2,4 & 3,5 & 4,4 & 4,4 \\
\hline$\underset{X}{\operatorname{IX}}$ & $\begin{array}{c}74,9-77,9 \\
=78,0\end{array}$ & $\begin{array}{l}2,4 \\
2,0\end{array}$ & - & $\begin{array}{l}1,0 \\
1,7\end{array}$ & $\begin{array}{l}1,1 \\
1,1\end{array}$ & $\begin{array}{l}1,1 \\
1,2\end{array}$ \\
\hline \multirow{2}{*}{ Total } & n. ${ }^{\circ}$ & 250 & 245 & 482 & 1.579 & 2.556 \\
\hline & $\%$ & 100,0 & 100,0 & 100,0 & 100,0 & 100,0 \\
\hline
\end{tabular}

T A B E L A I V

Dados trimestrais referentes aos comprimentos zoológicos $(\mathrm{cm})$ de cavala, Scomberomorus cavalla (Cuvier). Material capturado em frente ao município de Fortaleza (Estado do Ceará - Brasil), durante o período de janeiro a dezembro de 1970

\begin{tabular}{|c|c|c|c|c|}
\hline \multirow[b]{2}{*}{$\begin{array}{l}\text { Trimes- } \\
\text { tres }\end{array}$} & \multirow{2}{*}{$\begin{array}{l}\text { Número } \\
\text { de indi- } \\
\text { viduos } \\
\text { (n) }\end{array}$} & \multicolumn{3}{|c|}{ Comprimento zoológico $(\mathrm{cm})$} \\
\hline & & $\begin{array}{l}\text { valor } \\
\text { máximo }\end{array}$ & $\begin{array}{l}\text { valor } \\
\text { mínimo }\end{array}$ & $\begin{array}{l}\text { média } \\
\text { aritmética } \\
\frac{(\mathrm{x})}{}\end{array}$ \\
\hline I & 1.220 & 115,8 & 39,2 & 72,3 \\
\hline II & 1.240 & 124,0 & 50,4 & 68,6 \\
\hline III & 859 & 131,5 & 46,3 & 70,0 \\
\hline IV & 1.297 & 163,5 & 40,2 & 73,0 \\
\hline Total & 4.616 & 163,5 & 39,2 & 71,0 \\
\hline
\end{tabular}

'T A B E L A V

Dados trimestrais referentes aos comprimentos zoologicos $(\mathrm{cm})$ de serra, Scomberomorus maculatus (Mitchill). Material capturado em frente ao municipio de Fortaleza (Estado do Ceará - Brasil), durante o periodo de janeiro a dezembro de 1970 .

\begin{tabular}{|c|c|c|c|c|}
\hline \multirow{2}{*}{$\begin{array}{c}\text { Trimes- } \\
\text { tres }\end{array}$} & \multirow{2}{*}{$\begin{array}{l}\text { Número } \\
\text { de indi- } \\
\text { viduos } \\
(\mathrm{n})\end{array}$} & \multicolumn{3}{|c|}{ Comprimento zoológico $(\mathrm{cm})$} \\
\hline & & $\begin{array}{l}\text { valor } \\
\text { máximo }\end{array}$ & $\begin{array}{l}\text { valor } \\
\text { mínimo }\end{array}$ & $\begin{array}{l}\text { média } \\
\text { aritmética } \\
(\overline{\mathrm{x}})\end{array}$ \\
\hline $\begin{array}{l}\text { I } \\
\text { II } \\
\text { III } \\
\text { IV }\end{array}$ & $\begin{array}{r}250 \\
245 \\
482 \\
1.579\end{array}$ & $\begin{array}{l}97,8 \\
74,2 \\
93,8 \\
88,2\end{array}$ & $\begin{array}{l}36,9 \\
34,4 \\
33,9 \\
30,9\end{array}$ & $\begin{array}{l}58,1 \\
49,4 \\
52,6 \\
55,9\end{array}$ \\
\hline Total & 2.556 & 97,8 & 30,9 & 54,7 \\
\hline
\end{tabular}


T A B E L A V I

Dados de pescarias controladas de cavala, Scomberomorus cavalla (Cuvier), e de serra, Scomberomorus maculatus (Mitchill), realizadas por embarcaçōes que operaram em frente ao município de Fortaleza (Estado do Ceará - Brasil), durante o período de janeiro a dezembro de 1970 .

\begin{tabular}{|c|c|c|c|c|c|c|c|c|c|}
\hline \multirow{2}{*}{$\begin{array}{c}\text { Trimes- } \\
\text { tres }\end{array}$} & \multirow{2}{*}{$\begin{array}{l}\text { Número } \\
\text { de pes- } \\
\text { carias }\end{array}$} & \multirow{2}{*}{$\begin{array}{c}\text { Número } \\
\text { de } \\
\text { anzóis }\end{array}$} & \multirow{2}{*}{$\begin{array}{l}\text { Anzóis } \\
\text { por } \\
\text { pescaria }\end{array}$} & \multicolumn{2}{|c|}{$\begin{array}{l}\text { Individuos } \\
\text { capturados }\end{array}$} & \multicolumn{4}{|c|}{$\begin{array}{l}\text { Freqüências absolutas dos tipos de } \\
\text { iscas usadas }\end{array}$} \\
\hline & & & & cavala & serra & xira & sardinha & traíra $(\mathrm{s})$ & outros peixes \\
\hline $\begin{array}{l}\text { I } \\
\text { II } \\
\text { III } \\
\text { IV }\end{array}$ & $\begin{array}{l}529 \\
542 \\
662 \\
568\end{array}$ & $\begin{array}{l}4.519 \\
4.605 \\
5.408 \\
4.516\end{array}$ & $\begin{array}{l}8,5 \\
8,5 \\
8,2 \\
8,0\end{array}$ & $\begin{array}{r}1.008 \\
825 \\
505 \\
1.331\end{array}$ & $\begin{array}{r}1.381 \\
972 \\
759 \\
7.075\end{array}$ & $\begin{array}{l}301 \\
257 \\
292 \\
154\end{array}$ & $\begin{array}{r}206 \\
127 \\
77 \\
255\end{array}$ & $\begin{array}{r}4 \\
50 \\
62 \\
39\end{array}$ & $\begin{array}{r}18 \\
108 \\
231 \\
120\end{array}$ \\
\hline Total & 2.301 & 19.048 & 8,3 & 3.669 & 10.187 & 1.004 & 665 & 155 & 477 \\
\hline
\end{tabular}

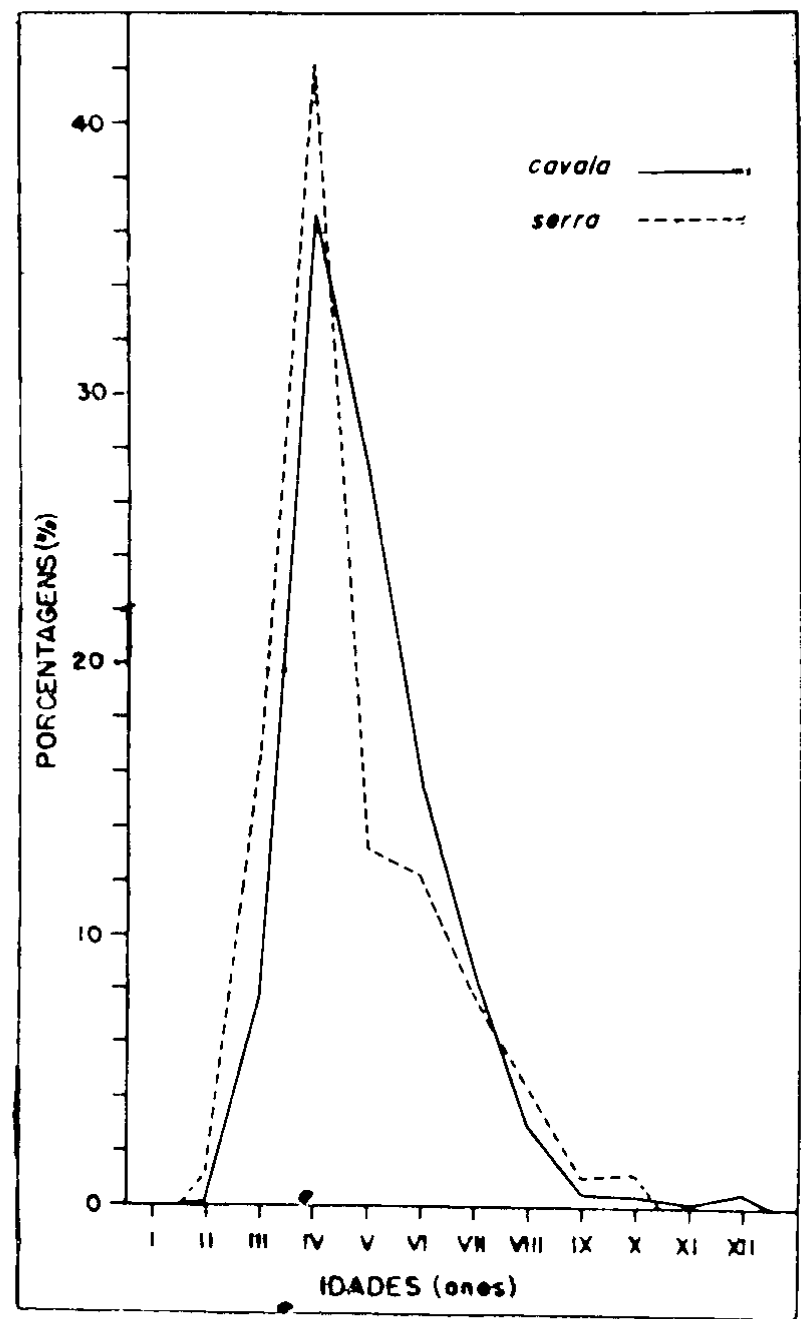

Figura 1 - Freqüências relativas de individuos das espécies Scomberomorus cavalla (Cuvier) e Scomberomorus maculatus (Mitchill), por grupos de idades, no conjunto geral das amostras. Material capturado em frente ao municipio de Fortaleza (Estado do Ceará - Brasil), no periodo de janeiro a dezembro de 1970 .

(1967), para a cavala e a serra, respectivamente.

O controle das pescarias foi efetuado diariamente, na praia de Mucuripe, logo após a chegada das embarcações selecionadas.
T A B E L A V I I

Indices trimestrais de captura por pescaria e de densidade relativa (captura por 100 anzóis/dia) de cavala, Scomberomorus cavalla (Cuvier), e de serra, Scomberomorus maculatus (Mitchill), na área de Fortaleza (Estado do Ceará - Brasil), durante o período de janeiro a dezembro de 1970 .

\begin{tabular}{|c|c|c|c|c|}
\hline \multirow{3}{*}{ Trimestres } & \multirow{2}{*}{\multicolumn{2}{|c|}{$\begin{array}{l}\text { Número de indiví- } \\
\text { duos capturados } \\
\begin{array}{c}\text { número de } \\
\text { pescarias }\end{array}\end{array}$}} & \multirow{2}{*}{\multicolumn{2}{|c|}{$\begin{array}{l}\text { Densidade } \\
\text { relativa* } \\
\text { (captura por } 100 \\
\text { anzóis/dia) }\end{array}$}} \\
\hline & & & & \\
\hline & cavala. & serra & cavala & serra \\
\hline I & 1,9 & 2,6 & 22,3 & 30,6 \\
\hline II & 1,5 & 1,8 & 17.9 & 21,1 \\
\hline III & 0,8 & 1,1 & 9,3 & 14,0 \\
\hline IV & 2,3 & 12,5 & 29,5 & 156,7 \\
\hline Total & 1,6 & 4,4 & 19,3 & 53,5 \\
\hline
\end{tabular}

* Deixaram de ser consideradas as outras espécies capturadas.

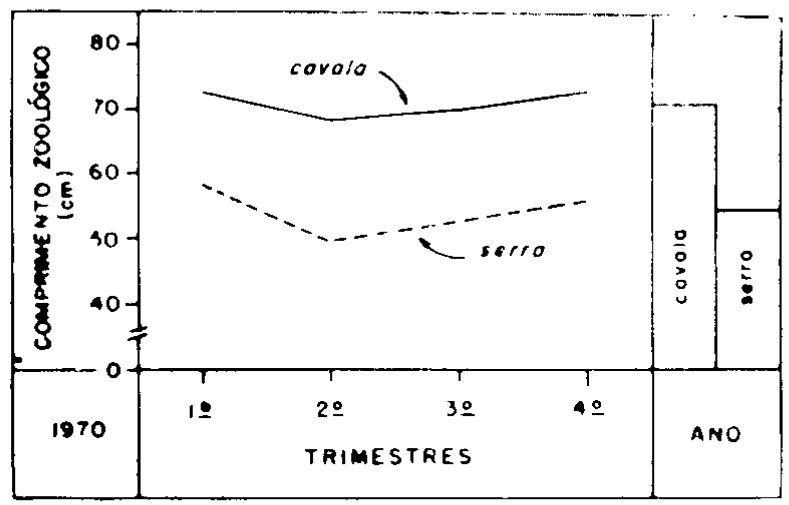

Figura 2 - Distribuicão das médias aritméticas ü comprimentos zoológicos de indivíduos das espécies Scomberomorus cavalla (Cuvier) e Scomberomoru" maculatus (Mitchill), correspondentes aos diversos conjuntos trimestrais de amostras. Material capturado em frente ao município de Fortaleza (Estado do Ceará - Brasil), no período de janeiro a dezembro de 1970 .

De cada pescaria realizada anotamos, principalmente o número de indivíduos capturados de cavala e de serra, o número de anzóis 


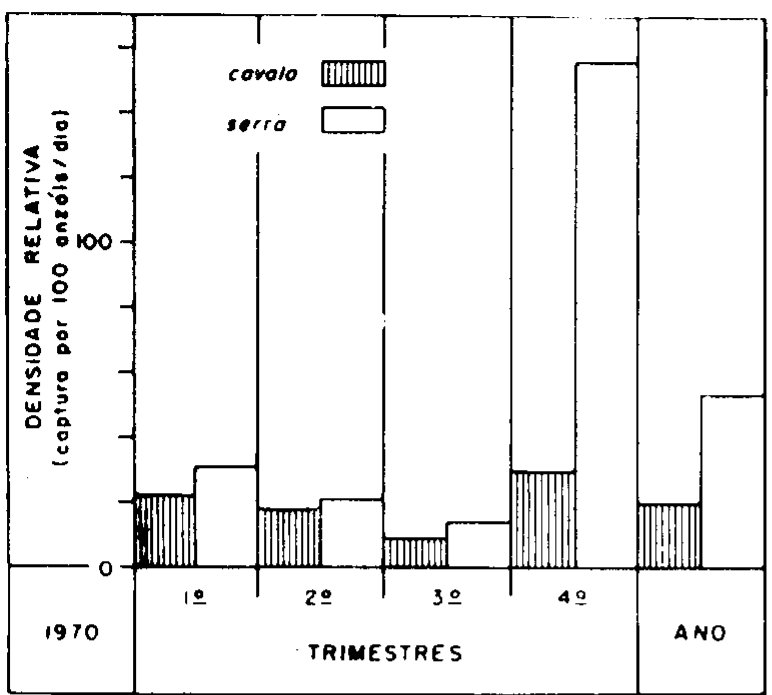

Figura 3 - Distribuição dos indices trimestrais de densidade relativa (captura por 100 anzóis/dia) das espécies Scomberomorus cavalla (Cuvier) e Scomberomorus maculatus (Mitchill). Material capturado em frente ao município de Fortaleza (Estado do Ceará - Brasil), no período de janeiro a dezembro de 1970 .

empregados e o tipo de isca usado, cujos dados foram agrupados por trimestres.

Os índices trimestrais de densidade relativa, determinados para cada espécie, foram calculados para 100 anzóis/dia.

\section{DISCUSSÃO E CONCLUSÕES}

Segundo Albuquerque \& Bezerra (1968), a amostragem mínima mensal para a cavala e a serra, no Estado do Ceará, deve ser de 400 e 300 indivíduos, respectivamente. Sendo assim, no tocante à cavala, tal exigência foi atendida nos meses de janeiro, fevereiro, abril, maio e no período de outubro a dezembro; e, para a serra somente no período de outubro a dezembro (tabela I) .

Para a cavala, os grupos de idades atingidos pela pesca, no conjunto geral das amostras, variaram entre os correspondentes a II e XII anos, concentrando-se entre os de III e VII anos, com máximo no de IV anos (tabela II , figura 1). Para a serra, estes grupos variaram entre os correspondentes a II e $\mathrm{X}$ anos, concentrando-se entre os de III e VII anos, com máximo no de IV anos (tabela III, figura 1) .

Nas pescarias da serra ao sul da Flórida (U.S.A.), os cinco primeiros grupos de idades são os que compõem os desembarques (Klima, 1959).

Apesar do número de indivíduos amostrados, de cada espécie em estudo, ter variado muito entre os trimestres, as médias aritméticas dos comprimentos zoológicos são muito próximas (tabelas IV e V; figura 2). Para a cavala, a maior destas médias foi a do quarto trimestre, quando atingiu o valor de $73,0 \mathrm{~cm}$, e a menor correspondeu ao segundo trimestre, expressa pelo valor de $68,6 \mathrm{~cm}$. Em relação à serra, a maior destas médias foi a do primeiro trimestre, quando atingiu o valor de $58,1 \mathrm{~cm}$, e a menor correspondeu ao segundo trimestre, expressa pelo valor de $49,4 \mathrm{~cm}$.

Os totais de anzóis/dia empregados nas pescarias controladas, em geral, variaram em relação direta com as freqüências trimestrais das pescarias; as médias trimestrais de anzóis utilizados por pescarias se apresentaram mais ou menos constantes; a média anual de anzóis por pescaria foi de 8,3 anzóis (tabela VI) . Os tipos de anzóis mais utilizados, nas pescarias controladas, foram os de números 4 e 5 para a cavala, e os de números 6 e 7 para a serra.

As iscas usadas nas pescarias controladas foram as seguintes: xira - Haemulon aurolineatum Cuvier, sardinha - Opisthonema oglinum (Le Sueur) e traíra(s) - Trachinocephalus myops (Forster) e/ou Synodus intermedius (Agassiz), em ordem decrescente de importância, sendo também empregados outros pequenos peixes marinhos, em escala muito pequeno, podendo mesmo não serem tomados em consideração. As freqüências trimestrais dos tipos de isca usados nas pescarias, mostram que, em quase todos os trimestres, a xira predominou sobre os demais peixes (tabela VI) .

Nas pescarias controladas, somente foram consideradas as capturas da cavala e da serra ( tabela VI) .

Os valores encontrados para a relação número de indivíduos capturados/número de pescarias e para a densidade relativa - captura por 100 anzóis/dia (tabela VII, figura 3), evidenciam que a captura por pescarias depende da densidade relativa.

Os mais altos índices de densidade relativa da cavala e da serra corresponderam ao quarto trimestre.

\section{SUMMARY}

With this paper the Marine Sciences Laboratory on the Federal University of Ceará carries on a serial study on the fishery biology of the king mackerel, Scomberomorus cavalla (Cuvier), and Spanish mackerel, Scomberomorus maculatus (Mitchill), in the State of Ceará, Brazil.

The material was caught off Fortaleza, and landed at Mucuripe beach. It corresponds to samples of 238 days of fishing carried out during the period from January to December, 1970 , made by rafts and some motor boats. We sampled 4,616 king mackerels and 2,556 Spanish mackerels. We have also controlled 2,301 rafts fishings, in the same area and 
throughout the period from January to December, 1970 .

For the king mackerel the age groups caught ranged from II to XII years, being observed concentration between III and VII years, and maximum at the IV years.

For the Spanish mackerel, the age group: caught ranged from II to $\mathrm{X}$ years, being observed concentration between III and VII years, and maximum at the IV years.

In the several monthly set of samples, the arithmetic means of fork lengths corresponding to each one of the species, are very close. For the king mackerel the greatest among those arithmetic means corresponded to the fourth trimester, when reached the value of $73.0 \mathrm{~cm}$, and the smallest one was reported in the second trimester, expressed through the value of $68.6 \mathrm{~cm}$. For the Spanish mackerel, the greatest among those arithmetic means corresponded to the first trimester, when reached the value of $58.1 \mathrm{~cm}$, and the smallest one was reported in the second trimester, expressed through the value of $49.4 \mathrm{~cm}$.

The total number of hooks used in controlled fishing, in general, varied in direct relationship to the trimestral frequencies of fishings. The trimestral means of the number of hooks used in each fishing were more or less constant. The year mean of hooks in each fishing was 8.3 hooks.

The types of bait used in controlled fishings were the tomtate - Haemulon aurolineatum Cuvier, the Atlantic thread-herring - Opisthonema oglinum (Le Sueur), and snakefish - Trachinocephalus myops (Forster) and/or sand diver - Synodus intermedius (Agassiz), in decreasing order of importance, being also used other marine small fish, in less number.

In the controlled fishings were considered only the king mackerel and Spanish mackerel catches, reaching the totals of 3,669 king mackerels and 10,187 Spanish mackerels.

We have studied the relations number of caught individuals/number of fishing and number of individuals caught by 100 hooks day (relative density). The catches by fishing depended of the relative density for the king mackerel and Spanish mackerel corresponded to the fourth trimester.

\section{BIBLIOGRAFIA CONSULTADA}

Albuquerque, J. J. L. \& Bezerra, R. C. F. - 1968 - Sóbre a amostragem da cavala e da serra no Ceara (Pisces). Rev. Brasil. Biol., Rio de Janeiro, 28 (2): 141-145, 2 figs.

Costa, R. S. \& Paiva, M. P. - 1963 - Notas sòbre a pesca da cavala e da serra no Ceará - Dadós de 1962 . Arq. Est. Biol. Mar. Univ. Ceará, Fortaleza, 3 (1) : 17-26, 4 figs.

Costa, R. S. \& Paiva, M. P. - 1964 -- Notas sôbre a pesca da cavala e da serra no Ceará - Dados de 1963. Arq. Est. Biol. Mar. Univ. Ceará, Fortaleza, 4 (2) : 71-81, 5 figs.

Costa, R. S. \& Paiva, M. P. - 1965 - Notas sōbre a pesca da cavala e da serra no Ceará - Dados de 1964. Arq. Est. Biol. Mar. Univ. Ceará, Fortaleza, 5 (2) : 93-101, 5 figs.

Costa, R. S. \& Paiva, M. P. -- 1966 - Notas sôbre a pesca da cavala e da serra no Ceará - Dados de 1965. Arq. Est. Biol. Mar. Univ. Fed. Ceará, Fortaleza, 6 (2) : 195-204, 4 figs.

Costa, R. S. \& Paiva, M. P. - 1967 - Notas sôbrc a pesca da cavala e da serra no Ceará -Dados de 1966. Arq. Est. Biol. Mar. Univ. Fed. Ceará, Fortaleza, 7 (2) : 181-190, 4 figs.

Costa, R. S. \& Paiva, M. P. - 1968 - Notas sôbre a pesca da cavala e da serra no Ceará - Dados de 1967. Arq. Est. Biol. Mar. Univ. Fed. Ceará, Fortaleza, 8 (2) : 125-131, 3 figs.

Costa, R. S. \& Paiva, M. P. - 1969 - Notas sôbre a pesca da cavala e da serra no Ceará - Dados de 1968. Arq. Ciên. Mar, Fortaleza, 9 (1) : 89-95, 3 figs.

Costa, R. S. \& Paiva, M. P. - $1970-$ Notas sôbre a pesca da cavala e da serra no Ceará - Dados de 1969. Arq. Ciên. Mar, Fortaleza, 10 (2) : 147-152, 3 figs.

Klima, E. F. - 1959 - Aspects of the biology and fishery for Spanish mackerel, Scomberomorus maculatus (Mitchill), of Southern Florida. State of Florida Board of Conservation Technical Series, Coral Gables, (27) : 1-39, 17 figs.

Nomura, H. - 1967 - Dados biológicos sôbre a serra, Scomberomorus maculatus (Mitchill), das águas cearenses. Arq. Est. Biol. Mar. Univ. Fed. Ceurá, Fortaleza, 7 (1) : 29-39, 4 figs.

Nomura, H. \& Rodrigues, M. S. S. - 1967 - Biological notes on king mackerel, Scomberomorus cavalla (Cuvier), from northeastern Brazil. Arq. Est. Biol. Mar. Univ. Fed. Ceará, Fortaleza, 7 (1) : 79-85, 4 figs. 